\title{
The immediate effects of foot pressure based insole on ankle dorsiflexion range of motion, postural sway, and muscle activation in healthy adults with genu varum
}

\author{
Hye-Lim Chun ${ }^{a}$, Byoung-Hee Lee ${ }^{b}$ \\ ${ }^{a}$ Department of Physical Therapy, Health and Welfare Graduate School, Sahmyook University, Seoul, Republic of Korea \\ ${ }^{b}$ Department of Physical Therapy, College of Health Science and Social Welfare, Sahmyook University, Seoul, Republic of Korea
}

Objective: The purpose of this research was to determine the immediate effects of wearing a foot-pressure-based insole (FPBI) on ankle dorsiflexion range of motion (DFROM), postural sway, and muscle activation in healthy individuals with genu varum. Design: Cross-sectional study.

Methods: This study was conducted on thirteen adults, with six male and seven females subjects. The mean age was 24.08 years. Foot pressure was measured to apply the FPBI and the weight bearing lunge test was performed with the application of a flat insole (FI) and FPBI. Examination was randomly performed in four conditions to measure both postural sway and muscle activation. All participants applied both the FI and FPBI with four conditions. The four conditions were as follows: 1) Romberg test posture with eyes closed, 2) Romberg test posture with eyes opened, 3) dominant single leg standing with eyes opened, and 4) non-dominant single leg standing with eyes opened.

Results: For ankle DFROM between the FI and FPBI, a significant increase was observed in both the dominant and non-dominant leg $(p<0.05)$. For postural sway between the FI and FPBI in the Romberg test posture with eyes closed and dominant single leg standing with eyes opened conditions, a significant decrease was observed $(p<0.05)$. However, the postural sway between FI and FPBI in the Romberg test posture with eyes opened and non-dominant single leg standing with eyes opened, no significant decrease was observed. Also, there were no significant effects on muscle activation between the application of the FI and FPBI. Conclusions: The result showed that FPBI immediately improved ankle DFROM and postural sway. It seems that FPBI may improve genu varum in healthy individuals with genu varum.

Key Words: Ankle dorsiflexion range of motion, Genu varum, Orthotic devices, Postural balance

\section{Introduction}

Genu valgum and genu varum are commonly reported as knee joint deformities [1]. Genu varum has been previously shown to increase the risk of osteoarthritis [2] and genu varum is common in osteoarthritis, because knee osteoarthritis more often affects the medial than the lateral compartment [3]. In bilateral standing with normal knee alignment, the weight-bearing line of the lower extremity passes through the center of the knee joint, thus the weight is distributed to the middle of the knee joint $[4,5]$. But in genu varum, the weight-bearing line is shifted from the middle to the medial part of the knee joint and the compressive forces over the medial part of the knee joint is increased [6].

A lateral wedge insole has been commonly used for genu varum for osteoarthritis [7-9] because it reduce pain [10] and knee adduction moment [11]. It also improves functions involved in physical activity, such as walking distance [7] and static balance [12]. It is also low-cost, and low-burden on self-management for managing genu varum with osteo-

Received: 20 November, 2018 Revised: 17 December, 2018 Accepted: 17 December, 2018

Corresponding author: Byoung-Hee Lee (ORCID https://orcid.org/0000-0001-9766-6068)

Department of Physical Therapy, College of Health Science and Social Welfare, Sahmyook University, 815 Hwarang-ro, Nowon-gu, Seoul 01795 , Republic of Korea

Tel: 82-2-3399-1634 Fax: 82-2-3399-1639 E-mail: 3679@syu.ac.kr

(c) This is an Open-Access article distributed under the terms of the Creative Commons Attribution Non-Commercial License (http://creativecommons.org/licenses/ by-nc/4.0) which permits unrestricted non-commercial use, distribution, and reproduction in any medium, provided the original work is properly cited.

Copyright $\odot 2018$ Korean Academy of Physical Therapy Rehabilitation Science 
arthritis [13].

Generally, genu varum caused by supination of hindfoot and pronation of forefoot and midfoot affects the quality of postural control [14], and poor postural control was reported in supinated or pronated feet during single-leg stance [15]. This may change the ground reaction force within the frontal plane and change the center of pressure location on the plantar side of the foot [6], which may disturb the balance control strategy during physical activity [16]. Increased subtalar pronation or supination moment may change the gravitation torque force on the foot and create stress over the longitudinal arch of the foot and thus postural stability may be affected by foot type in both static and dynamic conditions [14]. Another study also suggested that foot posture may have an influence on dynamic and static postural control with eye closed in those with chronic ankle stability [17]. Therefore, genu varum may have an influence on ankle instability.

Ankle dorsiflexion range of motion (DFROM) is often a point of emphasis during the rehabilitation of lower extremity pathologies [18]. In subjects with chronic ankle instability, foot pressure may influence several of the postural and DFROM measurements [14,15,19]. Ankle DFROM influences dynamic balance in subjects with chronic ankle instability [20]. Also, Ankle DFROM can play an important role in determining ankle dorsiflexor strength in young adults [21]. Another study reported that plantar flexor muscle strength was related to postural stability in healthy subjects [22].

Genu varum is related to external rotation of the hip joint, as well as posterior pelvic tilt, rearfoot supination, and elevation of the medial longitudinal arch [23]. Therefore, this posture affects muscle activation and strength in the lower extremities. A previous study showed that the genu varum group had more muscle activity in the vastus medialis than in the vastus lateralis and rectus femoris [24]. The vastus medialis/vastus lateralis ratio was also significantly higher in with genu varum than in values for both genu valgum and normal knee alignment [25].

Previous studies only reported the effects of applying a lateral wedge for genu varum with ankle DFROM [26], postural sway [12], or muscle activation [24]. However, there are no studies that investigated the combination of ankle DFROM, postural sway, and muscle activation. Therefore, this study was designed to investigate the immediate effects of wearing a foot-pressure-based insole (FPBI) on ankle DFROM, postural sway, and muscle activation in healthy in- dividuals with genu varum.

\section{Methods}

\section{Participants}

Thirteen subjects who had genu varum from Sahmyook University (Seoul, Korea) participated in this study. The inclusion criteria for this study were those with genu varum (gap in the medial epicondyle of the knee $\geq 3 \mathrm{~cm}$ ) and age between 20 to 29 years. Subjects with ankle sprain within 3 months, history of neuro-musculo-skeletal diseases, history of trauma or fracture in the lower limbs during the last year, professional athletes, and other lower limb deformities were excluded from the study [16].

The present study was approved by the Sahmyook University Institutional Review Board (IRB No. 2-1040781AB-N-01-2017023HR). The objectives of the study and its requirements were explained to the subjects, and all participants provided written consent in accordance with the ethical principles of the Declaration of Helsinki.

\section{Procedures}

One hundred forty-two subjects from freshman to junior year who study at the Sahmyook University (Seoul, Korea) Physical Therapy department were recruited. There were twenty-seven subjects with genu varum. There were twenty-seven subjects with genu varum but those who had an ankle sprain within 3 months had to be excluded, leaving a total of thirteen subjects. They were examined based on the inclusion and exclusion criteria, and informed consent regard to the examination procedure and duration was obtained.

Foot pressure was measured to apply the FPBI. The weight-bearing lunge test (WBLT) was performed with application of a flat insole (FI) and FPBI that was the same shape as the shoes. Measurement of postural sway and muscle activation were randomly performed in the Romberg test posture with eyes opened, Romberg test posture with eyes closed, dominant single leg standing with eyes opened, and non-dominant single leg standing with eyes opened conditions with the same shape as the shoes. All participants had the application of both FI and FPBI for all four conditions.

\section{Experimental methods}

\section{Measurement of genu varum}

In this study, genu varum was measured to select participants. To evaluate the genu varum, the participants were asked to stand against the wall with their back, buttocks 
and both heels touching the wall. They were required to stand in an anatomical position bare-footed. Then the subjects were asked to put their feet together. A gap of more than $3 \mathrm{~cm}$ between the two medial knee epicondyles was considered as genu varum [16,27].

\section{Measurement of foot pressure}

In this study, foot pressure was measured with the Zebris platform (Zebris FDM 1.5, 2016; ZEBRIS Medical, Isny, Germany). Measurement was done in an anatomical position, facing the front, and feet pelvic-width apart, bare-footed. Foot pressure was measured three times to minimize error of measurement.

\section{Application of the FPBI}

Based on the foot pressure, a 3 or 8-mm lateral wedge, which was made of silicon, were selectively applied to all participants (Figure 1). For those with a high arch, we ap-

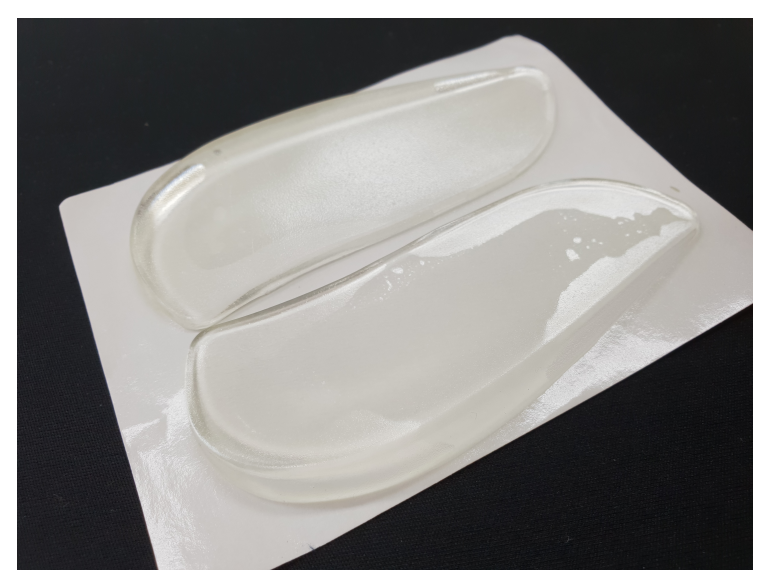

Figure 1. Foot pressure based insole.

(A)

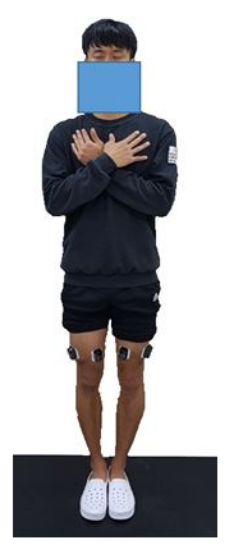

(B)

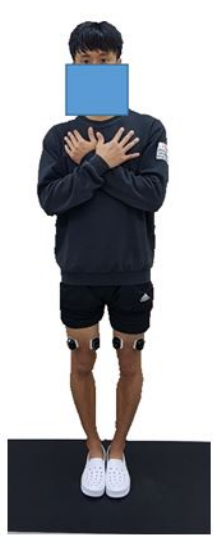

(C)

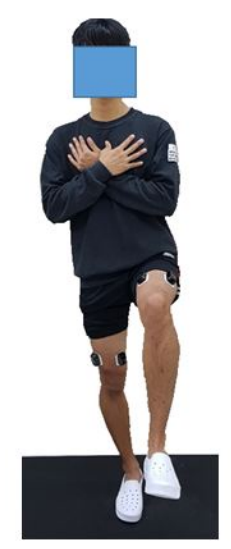

(D)

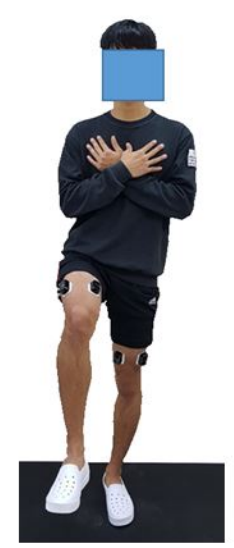

plied an 8-mm lateral wedge. For those with a normal arch, the $3 \mathrm{~mm}$ lateral wedge was applied. FI with non-wedged 1 mm thick, which was made of sponge, covered the whole shoe. It also covered the FPBI.

\section{Measurement of ankle DFROM}

In this study, ankle DFROM was measured with the WBLT $[28,29]$. The WBLT was matched between the second toe and center of the heel attached to the floor, and maintain the knee in vertical position on the wall. Distance between the second toe and the wall was recorded to measure ankle dorsiflexion angle indirectly [28]. The WBLT has a high reliability with a high intra-clinician reliability (ICC= 0.65-0.99) and inter-clinician reliability ( $\mathrm{ICC}=0.80-0.99)$ [18]. In this study, the inspector and assistant performed the measurements with both FI and FPBI application, and the average from three repeated measures was used to minimize error of measurement.

\section{Measurement of postural sway}

In this study, postural sway was measured with the Zebris platform (FDM 1.5; ZEBRIS Medical). Measurements were done with four positions in two conditions (Figure 2). The four positions were the Romberg test posture with eyes closed, Romberg test posture with eyes opened, dominant single leg standing with eyes open, and non-dominant single leg standing with eyes opened, and the two conditions were the FI and FPBI applications. Postural sway was measured three times for every condition and the average of three repeated measures was used to minimize error of measurement. The measurement was recorded for 20 seconds but data obtained from the middle 10 seconds was used for analysis. Thirty-second rest periods were provided between experi-

Figure 2. Measurement of postural sway and muscle activation. (A) Romberg test position with eye closed. (B) Romberg test position with eye opened. (B, C) Dominant single leg standing with eye opened. (D) Nondominant single leg standing with eye opened. 
ments [30].

\section{Measurement of muscle activation}

In this study, muscle activation was measured with the BIOS-mini (Biobrain Inc., Daejeon, Korea, 2016). Measurement was done with four positions in two conditions on the vastus lateralis muscle and medialis muscle of the dominant and non-dominant leg (Figure 2). The four positions were the Romberg test posture with eyes closed, Romberg test posture with eyes opened, dominant single leg standing with eyes open, and non-dominant single leg standing with eyes opened. In the dominant single leg standing condition, we measured muscle activation of the dominant leg. The two conditions were the FI and FPBI.

Before examining muscle activation, the electrode attachment site was cleaned with an alcohol swab, followed by removal of body hair to reduce skin resistance. Maximum voluntary isometric contraction (MVIC) was examined to calculate the root mean square (RMS). Muscle activation and MVIC was measured three times in every condition. The measurement was recorded for 20 seconds, and the middle of 10 seconds was used for analysis, and the average obtained from three repeated measures was used to minimize error of measurement.

\section{Measurement tools}

\section{Zebris FDM software and platform}

The 'ZEBRIS Win FDM' software was used for data collection and analysis where measurements were taken in standing position with the aid of a customary measurement platform (FDM 1.5; ZEBRIS Medical).

In this study, a platform was used to measure foot pressure and static postural sway of healthy individuals with genu varum with double leg standing and single leg standing positions. The platform size was $1,580 \mathrm{~mm}$ in length $\times 605$ $\mathrm{mm}$ in width $\times 21 \mathrm{~mm}$ in height. It incorporates 11,264 sensors on an area of 64 by $176 \mathrm{~cm}$, giving a resolution of ap-

Table 1. General characteristics of subject

$(\mathrm{N}=13)$

\begin{tabular}{lc}
\hline \multicolumn{1}{c}{ Characteristic } & Experimental group \\
\hline Gender (male/female) & $13(6 / 7)$ \\
Age $(\mathrm{y})$ & $24.08(3.01)$ \\
Height $(\mathrm{cm})$ & $167.82(9.73)$ \\
Weight $(\mathrm{kg})$ & $58.42(9.96)$ \\
Body mass index $\left(\mathrm{kg} / \mathrm{m}^{2}\right)$ & $20.64(2.30)$ \\
\hline
\end{tabular}

Values are presented as number only or mean (SD). proximately 4 sensors per square centimeter. All sensors were measured independently, the measuring frequency was $100 \mathrm{~Hz}$, and reported accuracy was within 5\%.

\section{Wireless 8-channel surface electromyography}

In this study, the BIOS-mini electrocardiogram (EKG), and surface electromyography (sEMG), which was completely wireless with an 8-channel measurement equipment, was used. EMG signal which analog signal was discrete digitizing to $1,000 \mathrm{~Hz}$ sampling frequency to possibly calculate index in data processing system, and digital resolving power is 24 bit.

After converting to digital signals, they were wirelessly sent to a personal computer to BioScan (2017; Biobrain Inc.), which is a professional EMG analysis program. Bioscan was applied with a 4-500 Hz Band-Pass Filter. After applying 60,120 , and $180 \mathrm{~Hz}$ Notch Filter to remove noise of power supply, the RMS index, which reflect muscle activation, was calculated.

\section{Statistical analysis}

All general characteristics of subjects were normally distributed. The PASW Statistics ver. 18.0 software (IBM Co., Armonk, NY, USA) was used for all statistical analysis. Results were presented as mean \pm standard deviation. Paired $\mathrm{t}$-test was used to compare the results between the FPBI and FI. For all tests, the level of statistical significance was set to 0.05 .

\section{Results}

\section{General characteristics of subject}

Thirteen subjects participated in this study. Demographic characteristics were shown in Table 1.

Table 2. Ankle dorsiflexion range of motion $(\mathrm{N}=13)$

\begin{tabular}{cccc}
\hline Side & FPBI & FI & $\mathrm{t}(p)$ \\
\hline $\begin{array}{c}\text { Dominant leg } \\
(\mathrm{cm})\end{array}$ & $14.73(2.93)$ & $12.62(3.04)$ & $5.628(<0.001)^{*}$ \\
$\begin{array}{c}\text { Non-dominant } \\
\text { leg }(\mathrm{cm})\end{array}$ & $14.27(3.61)$ & $13.05(2.92)$ & $3.666(0.003)^{*}$ \\
\hline
\end{tabular}

Values are presented as mean (SD). FPBI: foot pressure based insole, FI: flat insole. ${ }^{*} p<0.05$. 


\section{Ankle DFROM}

The results of ankle DFROM are shown in Table 2. In ankle DFROM between FI and FPBI, a significant increase was observed in both the dominant and non-dominant leg $(p<0.05)$.

\section{Postural sway}

The results of postural sway are shown in Table 3. In the $95 \%$ confidence ellipse area, center of pressure (COP) path, and COP average velocity between FI and FPBI in the Romberg posture with eyes closed, a significant decrease was observed $(p<0.05)$. In the $95 \%$ confidence ellipse area, COP path, and COP average velocity between FI and FPBI in Romberg posture with eyes opened, no significant decrease was observed ( $p>0.05)$. In the $95 \%$ confidence ellipse area, the COP path and COP average velocity between FI and FPBI in the dominant single leg standing position with eyes opened, a significant decrease was observed $(p<0.05)$. In the $95 \%$ confidence ellipse area, the COP path and COP average velocity between FI and FPBI in the non-dominant single leg standing position with eyes opened, no significant decrease was observed $(p>0.05)$.

\section{Muscle activation}

The results of muscle activation are shown in Table 4 . In muscle activation between FI and FPBI in the Romberg position with eyes closed condition, no significant decreases in vastus lateralis and medialis of the dominant leg and vastus lateralis and medialis of the non-dominant leg was observed $(p>0.05)$. In muscle activation between the FI and FPBI in the Romberg position with eyes opened condition, no significant decreases in vastus lateralis and medialis of dominant leg and vastus lateralis of non-dominant leg was observed $(p>0.05)$. In muscle activation between FI and FPBI in the Romberg position with eyes opened condition, no significant increases in vastus medialis of non-dominant leg was observed $(p>0.05)$.

In muscle activation between the FI and FPBI in the dominant single leg standing with eyes opened condition, no significant increases in the vastus lateralis and medialis of the dominant leg was observed $(p>0.05)$. In muscle activation between the FI and FPBI in the non-dominant single leg standing with eyes opened condition, no significant decreases in vastus lateralis and medialis of non-dominant leg was observed $(p>0.05)$.

\section{Discussion}

Genu varum increases the risk of osteoarthritis and it affects postural control. Therefore, this study was designed to investigate the effects of FPBI on ankle DFROM, postural

Table 3. Postural sway

$(\mathrm{N}=13)$

\begin{tabular}{|c|c|c|c|}
\hline Sway & FPBI & FI & $\mathrm{t}(p)$ \\
\hline \multicolumn{4}{|l|}{ Romberg with eye closed } \\
\hline $\mathrm{CEA}\left(\mathrm{mm}^{2}\right)$ & $409.96(178.22)$ & $625.72(375.71)$ & $2.756(0.017)^{*}$ \\
\hline $\mathrm{CPL}(\mathrm{mm})$ & $145.12(37.01)$ & $169.04(37.56)$ & $2.714(0.019)^{*}$ \\
\hline $\mathrm{CAV}(\mathrm{mm} / \mathrm{s})$ & $14.54(3.71)$ & $16.88(3.80)$ & $2.632(0.022)^{*}$ \\
\hline \multicolumn{4}{|l|}{ Romberg with eye opened } \\
\hline $\operatorname{CEA}\left(\mathrm{mm}^{2}\right)$ & $245.41(152.30)$ & $316.31(295.32)$ & $1.414(0.183)$ \\
\hline $\mathrm{CPL}(\mathrm{mm})$ & $90.00(25.49)$ & $93.69(33.24)$ & $0.826(0.425)$ \\
\hline $\mathrm{CAV}(\mathrm{mm} / \mathrm{s})$ & $8.95(2.55)$ & $9.38(3.37)$ & $1.019(0.328)$ \\
\hline \multicolumn{4}{|c|}{ Dominant single leg standing with eye opened } \\
\hline $\mathrm{CEA}\left(\mathrm{mm}^{2}\right)$ & $412.18(166.29)$ & $485.00(180.64)$ & $2.239(0.045)^{*}$ \\
\hline $\mathrm{CPL}(\mathrm{mm})$ & $273.17(85.15)$ & $299.27(86.51)$ & $2.533(0.026)^{*}$ \\
\hline $\mathrm{CAV}(\mathrm{mm} / \mathrm{s})$ & $27.47(8.58)$ & $30.00(8.64)$ & $2.437(0.031)^{*}$ \\
\hline \multicolumn{4}{|c|}{ Non-dominant single leg standing with eye opened } \\
\hline $\mathrm{CEA}\left(\mathrm{mm}^{2}\right)$ & $426.55(191.25)$ & $486.49(166.50)$ & $1.708(0.113)$ \\
\hline $\mathrm{CPL}(\mathrm{mm})$ & $268.03(53.05)$ & $291.12(68.57)$ & $1.516(0.155)$ \\
\hline $\mathrm{CAV}(\mathrm{mm} / \mathrm{s})$ & $26.82(5.21)$ & $29.17(3.80)$ & $1.529(0.152)$ \\
\hline
\end{tabular}

Values are presented as mean (SD).

FPBI: foot pressure based insole, FI: flat insole, CEA: 95\% confidence ellipse area, CPL: COP path length, CAV: COP average velocity. ${ }^{*} p<0.05$. 
Table 4. Muscle activation (electromyography)

$(\mathrm{N}=13)$

\begin{tabular}{|c|c|c|c|}
\hline Muscle & FPBI & FI & $\mathrm{t}(p)$ \\
\hline \multicolumn{4}{|c|}{ Romberg test posture with eye closed } \\
\hline Dominant VL (MVIC\%) & $3.84(3.76)$ & $5.34(5.64)$ & $1.897(0.082)$ \\
\hline Dominant VM (MVIC\%) & $6.34(8.92)$ & $6.52(7.99)$ & $0.171(0.867)$ \\
\hline Non-dominant VL (MVIC\%) & $3.91(3.64)$ & $6.06(7.90)$ & $1.631(0.129)$ \\
\hline Non-dominant VM (MVIC\%) & $8.91(10.97)$ & $9.34(14.33)$ & $0.143(0.888)$ \\
\hline \multicolumn{4}{|c|}{ Romberg test posture with eye opened } \\
\hline Dominant VL (MVIC\%) & $2.36(1.50)$ & $3.59(3.52)$ & $1.402(0.186)$ \\
\hline Dominant VM (MVIC\%) & $5.16(6.26)$ & $6.28(10.18)$ & $0.909(0.381)$ \\
\hline Non-dominant VL (MVIC\%) & $3.06(3.14)$ & $4.01(4.56)$ & $2.153(0.129)$ \\
\hline Non-dominant VM (MVIC\%) & $8.83(11.96)$ & $7.96(10.51)$ & $-1.556(0.052)$ \\
\hline \multicolumn{4}{|c|}{ Dominant single leg standing with eye opened } \\
\hline Dominant VL (MVIC\%) & $9.39(10.65)$ & $8.51(6.95)$ & $-0.430(0.675)$ \\
\hline Dominant VM (MVIC\%) & $12.42(12.55)$ & $10.77(9.92)$ & $-0.019(0.398)$ \\
\hline \multicolumn{4}{|c|}{ Non-dominant single leg standing with eye opened } \\
\hline Non-dominant VL (MVIC\%) & $11.99(17.26)$ & $13.72(21.06)$ & $1.297(0.219)$ \\
\hline Non-dominant VM (MVIC\%) & $19.31(26.61)$ & $20.03(25.49)$ & $0.166(0.871)$ \\
\hline
\end{tabular}

Values are presented as mean (SD).

FPBI: foot pressure based insole, FI: flat insole, VL: vastus lateralis, VM: vastus medialis, MVIC: maximum voluntary isometric contraction.

sway, and muscle activation in healthy adults with genu varum.

In this study, a significant increase in ankle DFROM between FI and FPBI was observed in both the dominant and non-dominant leg $(p<0.05)$. Previous studies reported that lateral wedged insoles produced small changes in ankle dorsiflexion in obese and healthy women [26], and there was a moderate correlation between ankle DFROM and dorsiflexor strength in healthy young adults [21]. It seems that FPBI may release the fascia around the ankle and foot, therefore, decrease the tension around the ankle and foot. Therefore, as the ankle DFROM increases, it is thought that flexibility of the muscle and fascia of the ankle and ankle dorsiflexor strength may have increased due to FPBI. As a result, FPBI may increase ankle DFROM.

Postural balance control was quantified by COP variables, which were velocity moment, total sway area, total sway length and confidence ellipse area [31]. In this study, the $95 \%$ confidence ellipse area between the FI and FPBI in the Romberg posture with eyes closed and dominant single leg standing position with eyes opened conditions was significantly decreased $(p<0.05)$. A previous study reported that the medio-lateral range of COP movement and the $95 \%$ confidence circle area of sway was significantly reduced after applying prefabricated orthotic insoles [32]. In this study, the COP path length between FI and FPBI in the Romberg posture with eyes closed and dominant single leg standing position with eyes opened was significantly decreased $(p<0.05)$. A previous study reported that examination during the eyes-closed task demonstrated an immediate reduction in the COP path length after insertion of the textured insoles compared to casual shoes alone in people with multiple sclerosis [33]. In this study, COP average velocity between FI and FPBI in the Romberg posture with eyes closed and dominant single leg standing position with eyes opened was significantly decreased $(p<0.05)$. Previous studies reported individuals in the supinated feet group of healthy young adults had significantly greater COP average speed [15], and mean values of COP total velocity during standing with and without insole were significantly decreased in subjects with flat feet [34]. Postural sway is affected by the visual, proprioception, and vestibular system. When healthy subjects stand on a firm surface, they rely on the visual system $10 \%$ for postural orientation [35]. Therefore, it is thought that the Romberg test posture with eyes opened condition might not be significant in this study. In this study, application time with FPBI was twenty minutes. Therefore, it is probably not affected to the non-dominant side of the leg. The main effects of lateral wedges were reductions of external knee adduction moment and of the knee adduction angular impulse $[3,36]$, and the lateral wedge insole shifted the weight bearing line from medial to the middle of the knee joint [6]. Therefore, this structural change may affect changes of muscle activation around the thigh muscle, and pelvic stability 
might be increased by co-contraction of the hamstring and quadriceps muscles. Lateral wedge insole may change the location of the navicular bone. Therefore, it may affect the elongation force of the tibialis posterior muscle. Also, a lateral wedge insole assists the ankle to change from supination to neutral position. Thus, sensory input may increase because contact area of the foot on the ground would be increased. Therefore, sensory input area may change from the lateral to the whole part of the foot. Consequently, it seems that FPBI may affect the $95 \%$ confidence ellipse area, $\mathrm{COP}$ path length, and COP average velocity. As a result, it is thought that FPBI affects postural sway in healthy subjects with genu varum.

In this study, for muscle activation between FI and FPBI in all conditions, no significant difference in all muscles of both dominant and non-dominant leg was observed. Previous studies reported that no statistical differences were observed in the vastus medialis and vastus lateralis for the EMG profiles between wedged and non-wedged conditions found in varum gonarthrosis [37], and standing on a wedge did not affect muscle activity of the proximal part [38]. Overall, the result of our study could support the findings from previous studies. It is considered that no significant effects appeared since this study only showed the immediate effects of applying FPBI. However, increased muscle tension can subsequently improve muscle strength, which can be expected to increase ankle stability [21].

The limitation of this study was as follows. First, we could not assess the long-term changes after applying FPBI because we only observed the immediate effects. Second, the number of subjects was small. Therefore, it is difficult for our results to be generalized to all healthy adults with genu varum. In future research studies, it would be necessary to address these limitations.

\section{Conflict of Interest}

The authors declared no potential conflicts of interest with respect to the authorship and/or publication of this article.

\section{References}

1. Kendall FP, McCreary EK, Provance PG, Rodgers M, Romani W. Muscles: testing and function with posture and pain. Philadelphia: Lippincott Williams \& Wilkins; 2005.

2. Sharma L, Song J, Felson DT, Cahue S, Shamiyeh E, Dunlop $\mathrm{DD}$. The role of knee alignment in disease progression and func- tional decline in knee osteoarthritis. JAMA 2001;286:188-95.

3. Matsumoto T, Hashimura M, Takayama K, Ishida K, Kawakami Y, Matsuzaki T, et al. A radiographic analysis of alignment of the lower extremities--initiation and progression of varus-type knee osteoarthritis. Osteoarthritis Cartilage 2015;23:217-23.

4. Johnson F, Leitl S, Waugh W. The distribution of load across the knee. A comparison of static and dynamic measurements. J Bone Joint Surg Br 1980;62:346-9.

5. Tetsworth K, Paley D. Malalignment and degenerative arthropathy. Orthop Clin North Am 1994;25:367-77.

6. Van Gheluwe B, Kirby KA, Hagman F. Effects of simulated genu valgum and genu varum on ground reaction forces and subtalar joint function during gait. J Am Podiatr Med Assoc 2005;95: 531-41.

7. Sattari S, Ashraf AR. Comparison the effect of 3 point valgus stress knee support and lateral wedge insoles in medial compartment knee osteoarthritis. Iran Red Crescent Med J 2011;13: 624-8.

8. Penny P, Geere J, Smith TO. A systematic review investigating the efficacy of laterally wedged insoles for medial knee osteoarthritis. Rheumatol Int 2013;33:2529-38.

9. Tokunaga K, Nakai Y, Matsumoto R, Kiyama R, Kawada M, Ohwatashi A, et al. Effect of foot progression angle and lateral wedge insole on a reduction in knee adduction moment. J Appl Biomech 2016;32:454-61.

10. Jones RK, Nester CJ, Richards JD, Kim WY, Johnson DS, Jari S, et al. A comparison of the biomechanical effects of valgus knee braces and lateral wedged insoles in patients with knee osteoarthritis. Gait Posture 2013;37:368-72.

11. Sawada T, Kito N, Yukimune M, Tokuda K, Tanimoto K, Anan $\mathrm{M}$, et al. Biomechanical effects of lateral and medial wedge insoles on unilateral weight bearing. J Phys Ther Sci 2016;28: 280-5.

12. Ganesan M, Lee YJ, Aruin AS. The effect of lateral or medial wedges on control of postural sway in standing. Gait Posture 2014;39:899-903.

13. Hunt MA, Takacs J, Krowchuk NM, Hatfield GL, Hinman RS, Chang R. Lateral wedges with and without custom arch support for people with medial knee osteoarthritis and pronated feet: an exploratory randomized crossover study. J Foot Ankle Res 2017;10:20

14. Cote KP, Brunet ME, Gansneder BM, Shultz SJ. Effects of pronated and supinated foot postures on static and dynamic postural stability. J Athl Train 2005;40:41-6.

15. Tsai LC, Yu B, Mercer VS, Gross MT. Comparison of different structural foot types for measures of standing postural control. J Orthop Sports Phys Ther 2006;36:942-53.

16. Samaei A, Bakhtiary AH, Elham F, Rezasoltani A. Effects of genu varum deformity on postural stability. Int J Sports Med 2012;33:469-73

17. Hogan KK, Powden CJ, Hoch MC. The influence of foot posture on dorsiflexion range of motion and postural control in those with chronic ankle instability. Clin Biomech (Bristol, Avon) 2016;38:63-7.

18. Powden CJ, Hoch JM, Hoch MC. Reliability and minimal detectable change of the weight-bearing lunge test: a systematic review. Man Ther 2015;20:524-32.

19. Hertel J. Functional instability following lateral ankle sprain. 
Sports Med 2000;29:361-71.

20. Basnett CR, Hanish MJ, Wheeler TJ, Miriovsky DJ, Danielson EL, Barr JB, et al. Ankle dorsiflexion range of motion influences dynamic balance in individuals with chronic ankle instability. Int J Sports Phys Ther 2013;8:121-8.

21. Guillén-Rogel P, San Emeterio C, Marín PJ. Associations between ankle dorsiflexion range of motion and foot and ankle strength in young adults. J Phys Ther Sci 2017;29:1363-7.

22. Bok SK, Lee TH, Lee SS. The effects of changes of ankle strength and range of motion according to aging on balance. Ann Rehabil Med 2013;37:10-6.

23. Neumann DA. Kinesiology of the musculoskeletal system: foundations for rehabilitation. St Louis, MO: Mosby; 2010.

24. Park S, Ko YM, Jang GU, Hwang YT, Park JW. A study on the differences of quadriceps femoris activities by knee alignment during isometric contraction. J Phys Ther Sci 2014;26:1685-8.

25. Sogabe A, Mukai N, Miyakawa S, Mesaki N, Maeda K, Yamamoto T, et al. Influence of knee alignment on quadriceps cross-sectional area. J Biomech 2009;42:2313-7.

26. Russell EM, Hamill J. Lateral wedges decrease biomechanical risk factors for knee osteoarthritis in obese women. J Biomech 2011;44:2286-91.

27. Magee DJ. Orthopedic physical assessment. Saint Louis: Saunders Elsevier; 2006.

28. Collins N, Teys P, Vicenzino B. The initial effects of a Mulligan's mobilization with movement technique on dorsiflexion and pain in subacute ankle sprains. Man Ther 2004;9:77-82.

29. Beazell JR, Grindstaff TL, Sauer LD, Magrum EM, Ingersoll $\mathrm{CD}$, Hertel J. Effects of a proximal or distal tibiofibular joint manipulation on ankle range of motion and functional outcomes in individuals with chronic ankle instability. J Orthop Sports Phys Ther 2012;42:125-34.

30. Abe Y, Sakamoto M, Nakazawa R, Shirakura K. Relationship between joint motion and acceleration during single-leg standing in healthy male adults. J Phys Ther Sci 2015;27:1251-6.

31. Sørensen RR, Jørgensen MG, Rasmussen S, Skou ST. Impaired postural balance in the morning in patients with knee osteoarthritis. Gait Posture 2014;39:1040-4

32. Bateni H. Changes of postural steadiness following use of prefabricated orthotic insoles. J Appl Biomech 2013;29:174-9.

33. Kalron A, Pasitselsky D, Greenberg-Abrahami M, Achiron A. Do textured insoles affect postural control and spatiotemporal parameters of gait and plantar sensation in people with multiple sclerosis? PM R 2015;7:17-25.

34. Tahmasebi R, Karimi MT, Satvati B, Fatoye F. Evaluation of standing stability in individuals with flatfeet. Foot Ankle Spec 2015;8:168-74.

35. Herdman SJ, Clendaniel RA. Vestibular rehabilitation. Philadelphia: FA Davis; 2014.

36. Kluge F, Krinner S, Lochmann M, Eskofier BM. Speed dependent effects of laterally wedged insoles on gait biomechanics in healthy subjects. Gait Posture 2017;55:145-9.

37. Giffin JR, Stanish WD, MacKinnon SN, MacLeod DA. Application of a lateral heel wedge as a nonsurgical treatment for varum gonarthrosis. J Prosthet Orthot 1995;7:23-8.

38. Ntousis T, Mandalidis D, Chronopoulos E, Athanasopoulos S. EMG activation of trunk and upper limb muscles following experimentally-induced overpronation and oversupination of the feet in quiet standing. Gait Posture 2013;37:190-4. 